\section{A Method to Detect and Characterize Ellipses Using the Hough Transform}

\author{
Nick Bennett, Robert Burridge, and \\ Naoki Saito, Member, IEEE
}

\begin{abstract}
In this paper we describe a new technique for detecting and characterizing ellipsoidal shapes automatically from any type of image. This technique is a single pass algorithm which can extract any group of ellipse parameters or characteristics which can be computed from those parameters without having to detect all five parameters for each ellipsoidal shape. Moreover, the method can explicitly incorporate any a priori knowledge the user may have concerning ellipse parameters. The method is based on techniques from Projective Geometry and on the Hough Transform. This technique can significantly reduce interpretation and computation time by automatically extracting only those features or geometric parameters of interest from images and making exact use of a priori information.
\end{abstract}

Index Terms-Hough Transform, ellipse detection, parameter estimation, projective geometry, feature extraction, computer vision.

\section{INTRODUCTION}

IMAGE characterization and interpretation is a very time-consuming job. Specifically, the problem of ellipse detection is often computationally demanding, since an ellipse is characterized by five parameters: center position coordinates; lengths of the major and minor axes; and the orientation of the major axis. Many wellstudied approaches have been used to address this problem:

1) probabilistic methods (see, for example, [8]), which attempt to obtain all five parameters while selectively sampling the image's edge data;

2) simulated annealing approaches (see, for example, [3]); and

3) multistage methods (see [13], [17], and [18]), which detect the parameters two or three at a time during each stage.

These methods usually combine both geometric insight and computational expertise in obtaining their results.

In this article, we offer a new tool for constructing and using parameter curves which translate image data (in this case, pairs of edges) into inferences about the possible ellipses present in the image. We present a single-stage method which can obtain any combination of ellipse parameters or any combination of characteristics which can be computed from those parameters. For example, our method can, in a single pass:

- obtain area and orientation information,

- obtain area and center position, or

- obtain minor axis length versus orientation.

Moreover, any a priori information (such as the maximum area, maximum eccentricity, or minimum minor axis length) can be explicitly incorporated into determining the endpoints of these parameter curves.

The key to our method is obtaining a parameterization for the

- N. Bennett and R. Burridge are at Schlumberger-Doll Research, Old Quarry Road, Ridgefield, CT 06877 USA.

E-mail: \{bennett; burridge\}@ridgefield.sdr.slb.com.

- N. Saito is now at the Department of Mathematics, University of California at Davis.E-mail: saito@math.ucdavis.edu.

Manuscript received 9 Sept. 1997; revised 23 Feb. 1999. Recommended for acceptance by $\mathrm{H}$. Keshavan.

For information on obtaining reprints of this article, please send e-mail to: tpami@computer.org, and reference IEEECS Log Number 108074. family of ellipses which are tangent to two line segments. From this parameterization, we can compute lower-dimensional parameter curves which describe any combination of parameters and characteristics of this family. We use these lower-dimensional curves to build single-stage Hough Transforms and to obtain these characterizations of any ellipse field.

We would propose that the parameter curve methodology detailed in this paper be combined with the many excellent computational strategies available in the literature in an object-oriented framework, so that users and researchers can mix together the ingredients which will be most successful for solving their problem. Towards this end, we offer a copy of the software used in the experiments described below (see the Acknowledgment section).

We begin with our parameterization and then proceed to construct the resulting Hough Transforms. We demonstrate our capabilities in a sequence of examples using very straightforward computational strategies in our implementation.

\section{Fundamentals}

Let $P_{1}$ be the point $\left(x_{1}, y_{1}\right)$ and $P_{2}$ be the point $\left(x_{2}, y_{2}\right)$ with associated normal directions $\mathbf{N}_{1}=\left(\mathbf{p}_{1}, \mathbf{q}_{1}\right)$ and $\mathbf{N}_{2}=\left(\mathbf{p}_{2}, \mathbf{q}_{2}\right)$, which for definiteness we suppose point into the angular sector between the tangent lines containing the other point (see Fig. 1). That is,

$$
\begin{aligned}
& p_{1}\left(x_{2}-x_{1}\right)+q_{1}\left(y_{2}-y_{1}\right)>0 \text { and } \\
& p_{2}\left(x_{1}-x_{2}\right)+q_{2}\left(y_{1}-y_{2}\right)>0
\end{aligned}
$$

We start by writing the equation for the line $P_{1} P_{2}$ and the two tangents. $P_{1} P_{2}$ is given by

$$
L(x, y) \equiv\left|\begin{array}{ccc}
x & y & 1 \\
x_{1} & y_{1} & 1 \\
x_{2} & y_{2} & 1
\end{array}\right|=0 .
$$

This equation is clearly linear in $x$ and $y$ and is satisfied when $P$, namely, $(x, y)$, coincides with $P_{1}$ or $P_{2}$, so it represents the line $P_{1} P_{2}$.

The tangent lines at $P_{1}$ and at $P_{2}$ are given by

$$
\ell_{1}(x, y) \equiv p_{1}\left(x-x_{1}\right)+q_{1}\left(y-y_{1}\right)=0
$$

and

$$
\ell_{2}(x, y) \equiv p_{2}\left(x-x_{2}\right)+q_{2}\left(y-y_{2}\right)=0,
$$

respectively.

The functions $L, \ell_{1}$, and $\ell_{2}$ are each linear in $x$ and $y$, so for given constant $\lambda$,

$$
C(x, y) \equiv L^{2}(x, y)-\lambda \ell_{1}(x, y) \ell_{2}(x, y)=0
$$

is quadratic in $x, y$ and represents a conic section $C$. For $\lambda=0,(3)$ represents the (repeated) line $L=0$ ( $L$ for short), and for $\lambda=\infty$ the line pair $\ell_{1}(x, y)=0$ or $\ell_{2}(x, y)=0\left(\ell_{1}\right.$ and $\left.\ell_{2}\right)$. For other values of $\lambda$, $C$ passes through the intersection of these degenerate conics (see Fig. 2). However, this intersection consists of two pairs of coincident points, each with an associated tangent direction, so the conic touches $\ell_{1}$ at $P_{1}$ and $\ell_{2}$ at $P_{2}$ (see Fig. 3).

We may write $C$ in the form

$$
C(x, y)=a x^{2}+2 h x y+b y^{2}+2 g x+2 f y+c,
$$

where $a, b, c, f, g, h$ are linear functions of $\lambda$ and also depend on $x_{1}$, $y_{1}, p_{1}, q_{1}, x_{2}, y_{2}, p_{2}, q_{2}$.

Let us write (4) in the form

$$
\mathbf{x}^{\mathrm{T}} \mathbf{A} \mathbf{x}+2 \mathbf{f}^{\mathrm{T}} \mathbf{x}+\mathbf{c}=0,
$$

where $\mathbf{x}=(x, y)^{\mathrm{T}}$ and $\mathbf{f}=(\mathrm{g}, \mathrm{f})^{\mathrm{T}}$ and $\mathbf{A}$ is the matrix

$$
\mathbf{A}=\left(\begin{array}{ll}
a & h \\
h & b
\end{array}\right)
$$




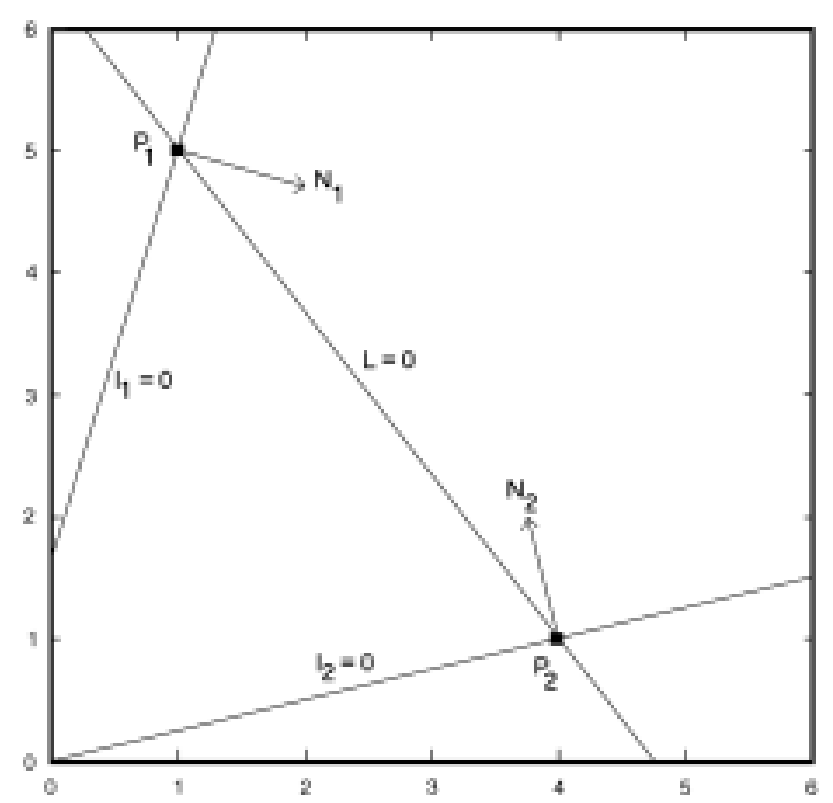

Fig. 1. $P_{1}=(1,5), P_{2}=(4,1), N_{1}=(1,3 / 10)$, and $N_{2}=(-1 / 4,1)$, with the corresponding lines $\ell_{1}, \ell_{2}$, and $L$.

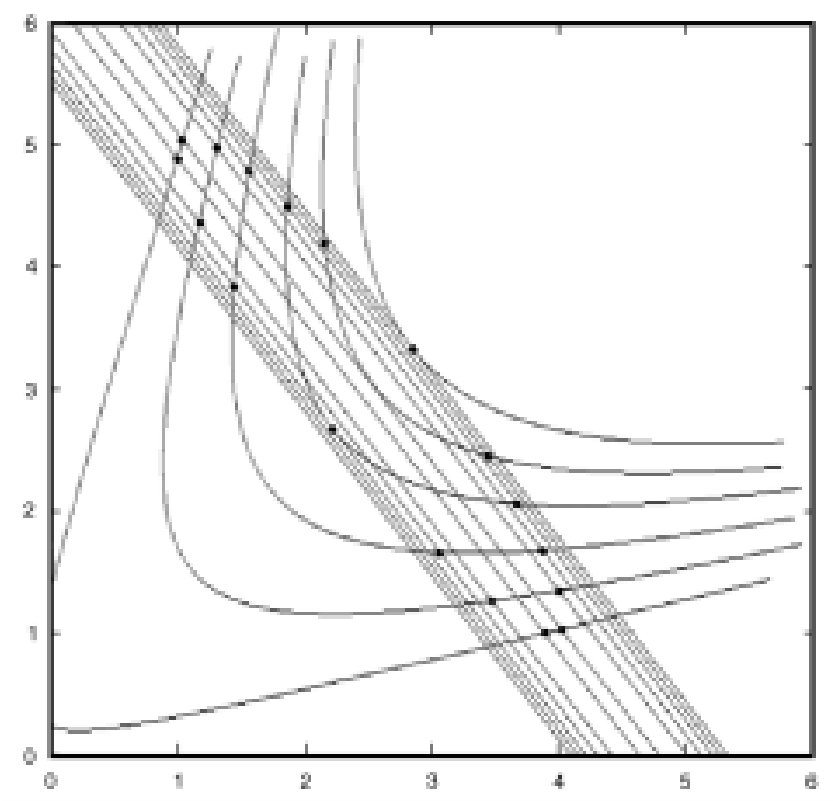

Fig. 2. Graphs of the equations $L(x, y)= \pm \sqrt{c}$ and $\ell_{1} \ell_{2}(x, y)=c$, for various values of $c$. The intersections of these curves are solutions to (3) for $\lambda=1$. Note that there may be zero, one, two, three, or four intersections for various $c$.

We then seek the center $\mathbf{x}_{0}=\left(x_{0}, y_{0}\right)^{\mathrm{T}}$ so that (5) may be written

$$
\left(\mathbf{x}-\mathbf{x}_{0}\right)^{\mathrm{T}} \mathbf{A}\left(\mathbf{x}-\mathbf{x}_{0}\right)=\mathrm{d},
$$

i.e.,

$$
\mathbf{x}^{\mathrm{T}} \mathbf{A} \mathbf{x}-2 \mathbf{x}_{0}^{\mathrm{T}} \mathbf{A} \mathbf{x}+\mathbf{x}_{0}^{\mathrm{T}} \mathbf{A} \mathbf{x}_{0}-\mathrm{d}=0 .
$$

Comparing (5) with (7), we find that

$$
\mathbf{A x}_{0}=-\mathbf{f} \text {, }
$$

giving

$$
\mathbf{x}_{0}=-\mathbf{A}^{-1} \mathbf{f}
$$

Then

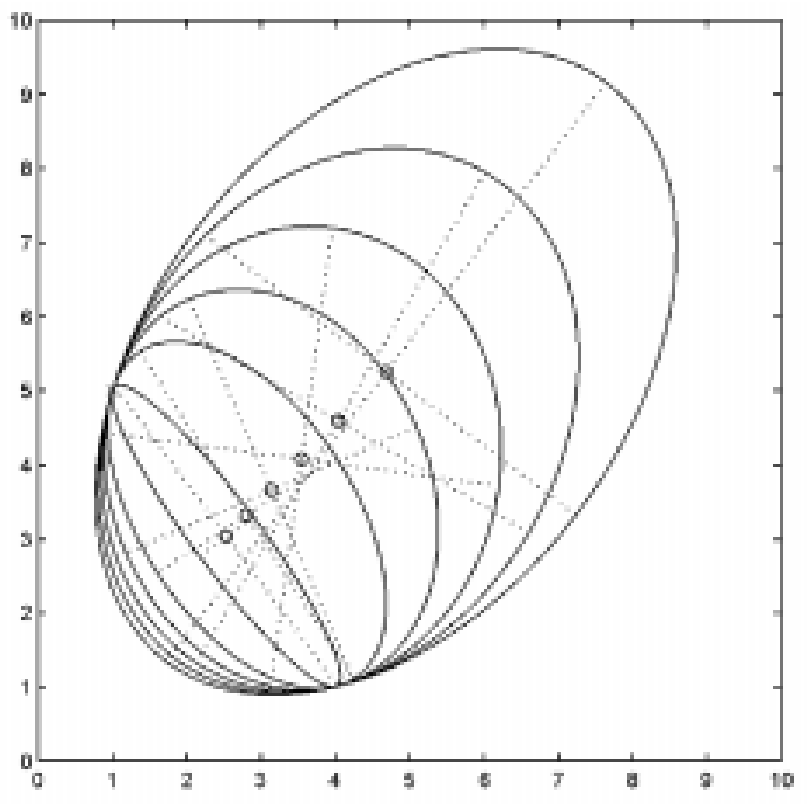

Fig. 3. Graphs of (3) for various values of $\lambda$ ranging from one to 39.

$$
\mathbf{x}_{0}^{\mathrm{T}} \mathbf{A} \mathbf{x}_{0}-\mathrm{d}=\mathbf{f}^{\mathrm{T}} \mathbf{A}^{-1} \mathbf{f}-\mathrm{d}=\mathrm{c},
$$

or

$$
\mathrm{d}=\mathbf{f}^{\mathrm{T}} \mathbf{A}^{-1} \mathbf{f}-\mathrm{c} .
$$

\subsection{The Range of $\lambda$ for Which the Conic Is an Ellipse}

Equation (7) represents a parabola if $\mathbf{A}$ is singular, since then the center $\mathbf{x}_{0}$ is at infinity. It represents an ellipse or a hyperbola or is vacuous if $(1 / d) \mathbf{A}$ is positive definite, indefinite, or negative definite.

Let us look more closely at the quantities $a, b, \ldots h$. From (2),

$$
\begin{gathered}
L(x, y)=\left(y_{1}-y_{2}\right) x+\left(x_{2}-x_{1}\right) y+x_{1} y_{2}-x_{2} y_{1}, \\
\ell_{1}(x, y)=p_{1} x+q_{1} y-p_{1} x_{1}-q_{1} y_{1}, \\
\ell_{2}(x, y)=p_{2} x+q_{2} y-p_{2} x_{2}-q_{2} y_{2} .
\end{gathered}
$$

It follows that

$$
\begin{aligned}
a(\lambda)= & \left(y_{1}-y_{2}\right)^{2}-\lambda p_{1} p_{2} \\
b(\lambda)= & \left(x_{2}-x_{1}\right)^{2}-\lambda q_{1} q_{2} \\
c(\lambda)= & \left(x_{1} y_{2}-x_{2} y_{1}\right)^{2}-\lambda\left(p_{1} x_{1}+q_{1} y_{1}\right)\left(p_{2} x_{2}+q_{2} y_{2}\right) \\
f(\lambda)= & \left(x_{1} y_{2}-x_{2} y_{1}\right)\left(x_{2}-x_{1}\right)+ \\
& \frac{1}{2} \lambda\left[q_{1}\left(p_{2} x_{2}+q_{2} y_{2}\right)+q_{2}\left(p_{1} x_{1}+q_{1} y_{1}\right)\right] \\
g(\lambda)= & \left(x_{1} y_{2}-x_{2} y_{1}\right)\left(y_{1}-y_{2}\right)+ \\
& \frac{1}{2} \lambda\left[p_{1}\left(p_{2} x_{2}+q_{2} y_{2}\right)+p_{2}\left(p_{1} x_{1}+q_{1} y_{1}\right)\right] \\
h(\lambda)= & \left(y_{1}-y_{2}\right)\left(x_{2}-x_{1}\right)-\frac{1}{2} \lambda\left(p_{1} q_{2}+p_{2} q_{1}\right)
\end{aligned}
$$

Let us now examine the signs of the terms $L^{2}$ and $\ell_{1} \ell_{2}$ in (3). $L^{2}$ is positive except on the line $L$, where it is zero. Because of (1), the product $\ell_{1} \ell_{2}$ is positive for $\mathbf{x}$ in the sector between $\ell_{1}$ and $\ell_{2}$ containing the segment $P_{1} P_{2}$. Hence, if $\lambda<0$, (3) is not satisfied for any $\mathbf{x}$ in that sector or in the opposite sector, where also $\ell_{1} \ell_{2}>0$. However, these sectors divide the plane into two separated domains. Thus, for $\lambda<0$, any conic touching $\ell_{1}$ and $\ell_{2}$ must have two separated components and is thus a hyperbola. Hence, we need only 


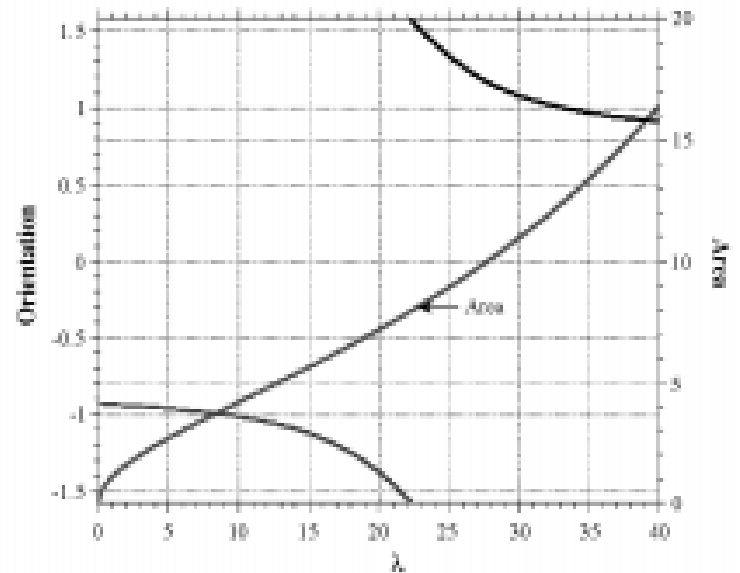

(a)

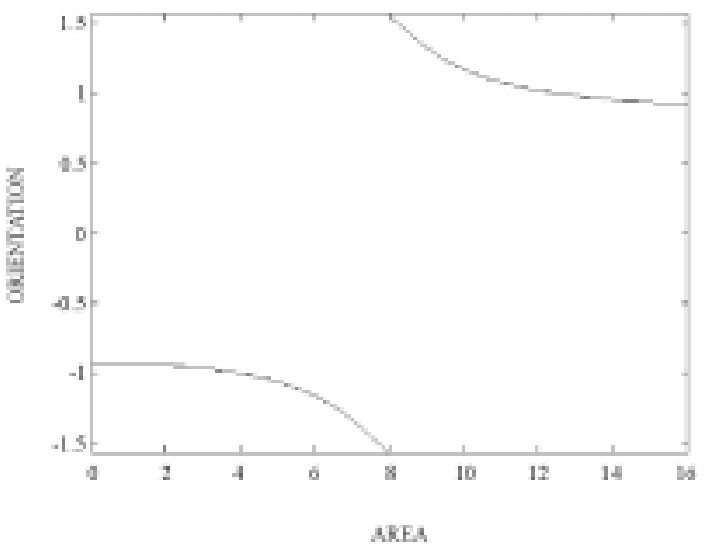

(b)

Fig. 4. (a) Graphs of area and orientation as functions of $\lambda$ computed from the edge data used in the previous figures. Note that the area curve has a vertical asymptote at $\lambda_{0}=93.27$. (b) Graph of the planar curve whose components give area and orientation as functions of $\lambda$.

consider positive values of $\lambda$. When $\lambda$ is small and positive, the conic is close to the line segment $P_{1} P_{2}$, to which it tends as $\lambda \rightarrow 0$. When $\lambda=0$, the matrix $\mathbf{A}$ is singular, but, as $\lambda$ increases, $\mathbf{A}$ at first becomes positive definite and then becomes singular (indefinite) again for a positive value $\lambda_{0}$ of $\lambda$. For $\lambda$ larger than $\lambda_{0}$, A becomes indefinite. This behavior corresponds to the center of the ellipse at first receding to infinity, so that the ellipse tends to a parabola for $\lambda=\lambda_{0}$, and then, for $\lambda>\lambda_{0}$, the conic becomes a hyperbola, but this time within the sectors for which $\ell_{1} \ell_{2}$ is positive. Thus, the range of $\lambda$ for which the conic $C$ of (3) is an ellipse is the interval $0<\lambda<$ $\lambda_{0}$. Hence, we must solve

$$
\operatorname{det}(\mathbf{A})=\left|\begin{array}{ll}
a & h \\
h & b
\end{array}\right|=\mathbf{0}
$$

for $\lambda$. One root is zero; the other is the required value $\lambda_{0}$ given by

$$
\lambda_{0}=\frac{4\left[p_{1}\left(x_{2}-x_{1}\right)+q_{1}\left(y_{2}-y_{1}\right)\right]\left[p_{2}\left(x_{1}-x_{2}\right)+q_{2}\left(y_{1}-y_{2}\right)\right]}{\left(p_{1} q_{2}-p_{2} q_{1}\right)^{2}} .
$$

Thus, the conic $C$ of (3) represents an ellipse if and only if $0<\lambda<\lambda_{0}$. For $\lambda$ in this range, one may calculate the coordinates of the center of the ellipse, $\left(x_{0}(\lambda), y_{0}(\lambda)\right)$, via (8). Note that $x_{0}(\lambda)$ and $y_{0}(\lambda)$ parameterize the line previously found in [18]. Observe that

$$
\left(x_{0}(\lambda), y_{0}(\lambda)\right)=\left(\frac{\mathcal{F}_{1}+\mathcal{F}_{2} \lambda}{\mathcal{F}_{3}+\mathcal{F}_{4} \lambda}, \frac{\mathcal{F}_{5}+\mathcal{F}_{6} \lambda}{\mathcal{F}_{3}+\mathcal{F}_{4} \lambda}\right)
$$

where the constants depend only on the image data. It is easy to show that $x_{0}(\lambda)$ and $y_{0}(\lambda)$ parameterize a line (one can even compute $d y / d x$ implicitly).

The lengths of the major and minor axes are

$$
\rho_{1}(\lambda)=1 / \sqrt{\kappa_{1}} \text { and } \rho_{2}(\lambda)=1 / \sqrt{\kappa_{2}}
$$

where $\kappa_{1}>\kappa_{2}$ are the eigenvalues of $\mathbf{A} / \mathbf{d}$ :

$$
\begin{aligned}
& \kappa_{1}=\frac{1}{d}\left(\frac{a+b}{2}-\sqrt{\frac{(a-b)^{2}}{4}+h^{2}}\right) \\
& \kappa_{2}=\frac{1}{d}\left(\frac{a+b}{2}+\sqrt{\frac{(a-b)^{2}}{4}+h^{2}}\right) .
\end{aligned}
$$

This leads to the usual formula for area as a function of $\lambda$ :

$$
\text { Area }=\frac{\pi|d|}{\sqrt{a b-h^{2}}}
$$

The orientation of the axes is given by

$$
\beta(\lambda)=\arctan \left(\frac{d \kappa_{2}-a}{h}\right)
$$

where the matrix $\mathbf{A}$ is given in (6) and where $a(\lambda), b(\lambda)$, and $h(\lambda)$ are given in (9). See Fig. 4 for example graphs of area and orientation as functions of $\lambda$.

For the interested reader, we cite [12] as a complete general reference for this material.

\section{Edge Detection Images}

The presence of ellipsoidal shapes is often revealed by discontinuities or edges in the image values, and there are several groups of well-studied edge detection techniques ([10], [11], [2], [7], and others). Useful surveys of these techniques can be found in [9] and in [19]. In an object-oriented framework, we can foresee any number of edge detection techniques being used successfully. Using an edge detection technique, we can compute a set of edge points (coordinates) at the boundaries of these ellipsoidal shapes, $P_{i}=\left(x_{i}, y_{i}\right)$, whose number depends on the length of the ellipse's boundary. Moreover, we can measure the slopes (or orientations) of the edge points by computing the edge gradients; we can simply add 90 degrees to the edge gradient direction to obtain the edge orientation, $\eta_{i}$. In addition, we can measure the strength or magnitude of the edge gradient, $m_{i}$. In this paper, we have employed the wellknown Canny edge detector using an implementation of the Robot Vision Group in the Department of Artificial Intelligence at the University of Edinburgh (also included with our software). This software produces an array of edge orientations and an array of edge magnitudes. We use both files as input into the Hough Transform processing described below.

\section{SOME SIMPLE EXPERIMENTS}

With the parameterization of Section 2 and our collection of edge data $\vec{e}_{i}=\left(P_{i}, \eta_{i}, m_{i}\right)$ in hand, we can construct Hough Transforms to answer a variety of sample questions concerning ellipse fields.

\subsection{Center-Finding Experiment}

Suppose we are interested in finding the centers of the ellipses in Fig. 5. The five ellipses have area 0.028, eccentricities around 2.5, and orientations $-\frac{\pi}{2}+k \frac{\pi}{6}$, for $k=1, \ldots, 5$. Suppose, for example, that we knew that the ellipses had ratios of major axis length to minor axis length less than 4.0. We shall see below how to incorporate this information explicitly in our implementation. As a first 


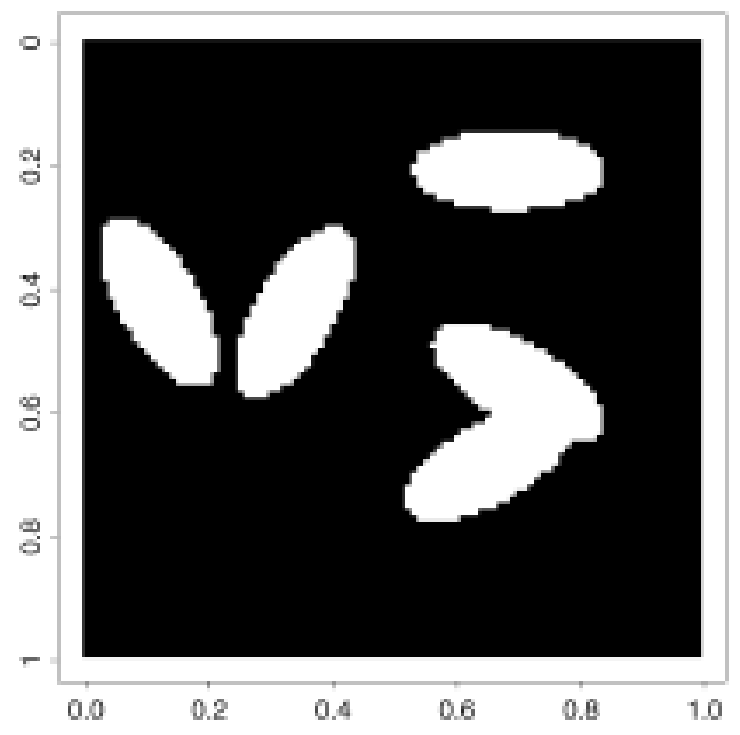

Fig. 5. An illustration of five intersecting ellipses with the same area.

step, we process the image in Fig. 5 using the Canny edge detector mentioned above.

In order to make use of the discussion in Section 2 for our collection of edge data, we set $q_{i}=1$ and $p_{i}=\tan \eta_{i}$. Note that for each pair of edges from which we will construct a parameter curve, we check the conditions in (1). If one of these conditions is not met, we use the normal vector $-\mathrm{N}_{\mathbf{k}}$ instead of $\mathrm{N}_{\mathbf{k}}$ in defining our curve. We then use the parameterizations in (11) to define the curve $\left(x_{0}(\lambda), y_{0}(\lambda)\right)$, which we have shown parameterizes the line previously found in [18]. When considering candidate ellipses (parameterized by $\lambda$ ) for voting during the Hough Transform step, we eliminate those with ratio of major axis length to minor axis length exceeding 4.0. That is, we register a vote for a candidate ellipse only if

$$
\operatorname{eccentricity}(\lambda)=\sqrt{\rho_{1}^{2}(\lambda)-\rho_{2}^{2}(\lambda)} / \rho_{1}(\lambda)<\frac{\sqrt{15}}{4},
$$

where $\rho_{1}$ and $\rho_{2}$ are defined in (12).

In order to obtain the most consistent estimates for the ellipse centers given the edge points, $\left\{\vec{e}_{i}=\left(x_{i}, y_{i}, \eta_{i}, m_{i}\right)\right\}$, we use the Hough Transform ([4], [5], [8]) which was originally developed to detect straight lines in images. This technique is essentially a "voting" or "evidence gathering" scheme: given a pair of data points from our collection $\vec{e}_{i}, \vec{e}_{j}$, vote for the center position of all

possible ellipses passing through $\left(x_{k}, y_{k}\right)$ with the slope $\tan \eta_{k}$ for $k$ $=i, j$, which agree with all of our a priori information, and then accumulate the votes from all data points to get the desired parameters. Our simple implementation proceeds as follows. We prepare the so-called "accumulator array" which is a discretized version of the two-dimensional parameter space for the ellipse center position. The array contents are initially set to zero. Then, for each pair of edges $\vec{e}_{i}, \vec{e}_{j}$ which both have sufficient magnitude,

$m_{i}, m_{j}>M$, and which are sufficiently close together, $\left|P_{i}-P_{j}\right|<B$ ( $M$ and $B$ are user controls in our software), we increment the array contents through which the curve determined by (11) and (15) passes. Thus, if there exists an ellipse in the image so that many pairs of edge points correspond to it, the corresponding curves will pass through a common point and that element/cell of the accumulator array accumulates many votes. Finally, we can select a maximum in the accumulator array to obtain the most consistent parameters representing that ellipse. There are a number of wellstudied additional techniques for improving this very simple

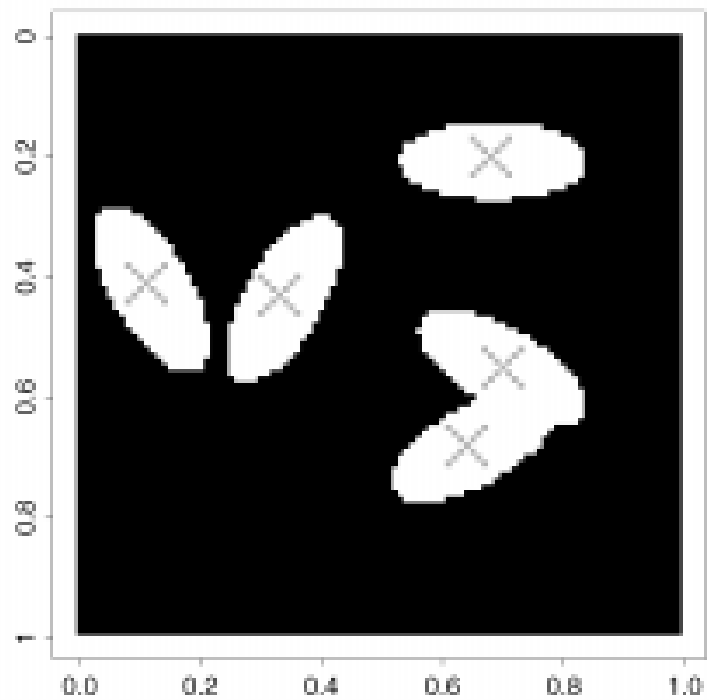

Fig. 6. An illustration of the top five ellipse centers found during sample experiment 1.

computational approach, but in this paper we focus on presenting our new parameterization and its uses as a single-pass method and as a means of explicitly incorporating a priori information. These additional methods include:

1) the "bow-tie" method to compensate for uncertainty in edge orientations, (see [5]);

2) the use of Average Shifted Histograms (see [14]) to compensate for problems in accurately estimating parameters due to the discretization of the parameter space; and

3) the many very recent techniques introduced in [6], [16], [15], [1], and [3].

These would all be potentially very valuable modules to include in an actual implementation but are beyond the scope of this correspondence.

In our example, we constructed a $100 \times 100$ accumulator array to discretize the center position parameters. We applied our Hough Transform for detecting center position to the edge data. The top five centers found by computing the top five local maxima in the accumulator array are shown in Fig. 6. The reader is invited to use the software we provide (see the Acknowledgment section) to repeat our experiment and see that, in this case, the a priori information had negligible effect on the result.

\subsection{Area- and Orientation-Finding Experiment}

Suppose we are interested in the areas and orientations of the ellipses contained in the unit square pictured in Fig. 5 after having completed the work of Section 4.1.

Using the edge angle and magnitude data pictured and a list of centers produced in Section 4.1, we construct our Hough Transform. For each pair of edges $\vec{e}_{i}, \vec{e}_{j}$, we use the parameterizations in (13) and (14) to define the curve

$$
\left(\operatorname{Area}(\lambda), \beta(\lambda) ; \vec{e}_{i}, \vec{e}_{j}\right), 0 \leq \lambda<\lambda_{0} .
$$

See Fig. 4 for an example graph of this curve. When considering candidate ellipses for voting during the Hough Transform step, we eliminate those whose centers, $\left(x_{0}(\lambda), y_{0}(\lambda)\right)$, are more than a distance $D$ (another user control in the software) from all of the ellipse centers given in our a priori information.

We constructed a $100 \times 100$ accumulator array to discretize the area and orientation parameters. We applied our Hough Transform for detecting area versus orientation to the edge data. The results obtained by incorporating and not incorpo- 


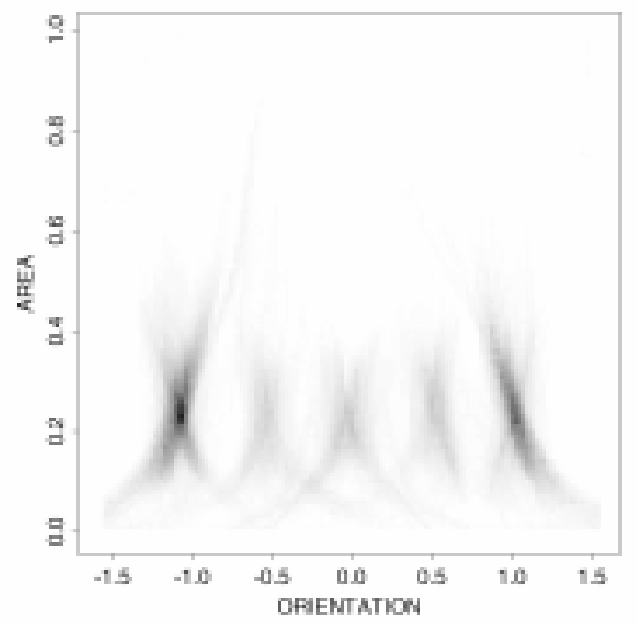

(a)

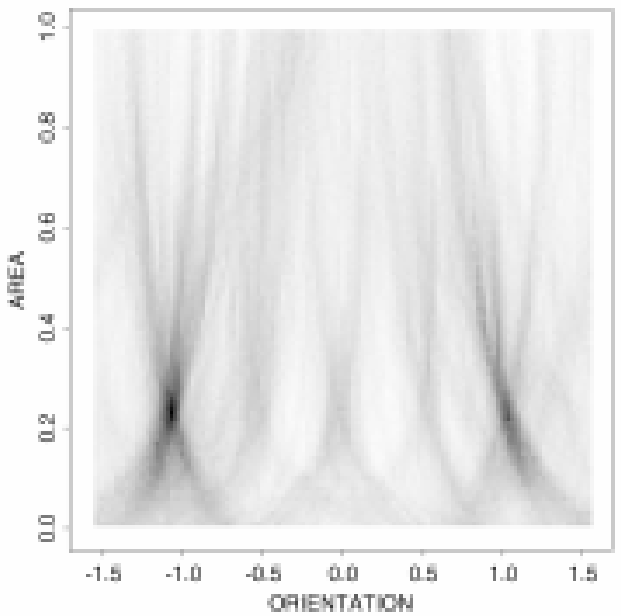

(b)

Fig. 7. Results of the area/orientation experiment performed with and without the a priori center information.

rating the a priori center position information are pictured in Fig. 7.

\subsection{Center Position and Area Experiment}

Suppose we are interested in computing the center positions and areas for the collection of ellipsoidal shapes in Fig. 8, another $100 \times 100$ image. We use the Canny edge detector technique to obtain the edge orientation and magnitude information.

\section{TABLE 1}

\section{SCORES FOR CENTER POSITION AND AREA EXPERIMENT}

\begin{tabular}{|c|c|c|c|c|c|c|}
\hline CALC $X_{n \mid}$ & TRLE $X_{0}$ & CALC $Y_{0}$ & TRUE $Y_{0}$ & CALC AREA & TRUE AREA & SCORE \\
\hline 0.333 & 0.313 & 0.13 .1 & $0.1 .1]$ & 0.036 .1 & 0.0317 & 121050 \\
\hline 0.838 & $0.8 \pm 0$ & 01.444 & 0.452 & 0.0354 & 0.0329 & 9790 \\
\hline 0.202 & 10.211 & 0.697 & 0.703 & $(0.0101$ & 0.0085 & $73: 10$ \\
\hline 0. .886 & $0.69 ?$ & 01.717 & $0 . \overline{7} 14$ & 0.01 .31 & 0.072 & 69870 \\
\hline 0.889 & 0.885 & $0.6+16$ & 0.051 & 0.02222 & 0.0195 & $49+70$ \\
\hline 0.889 & & 0.6416 & & 0.020 & & 44880 \\
\hline 0.323 & & 0.434 & & 0.027 & & 40800 \\
\hline $0.8 \div 88$ & & $0.4+4$ & & $0.02 \bar{\tau}$ & & $377+0$ \\
\hline
\end{tabular}

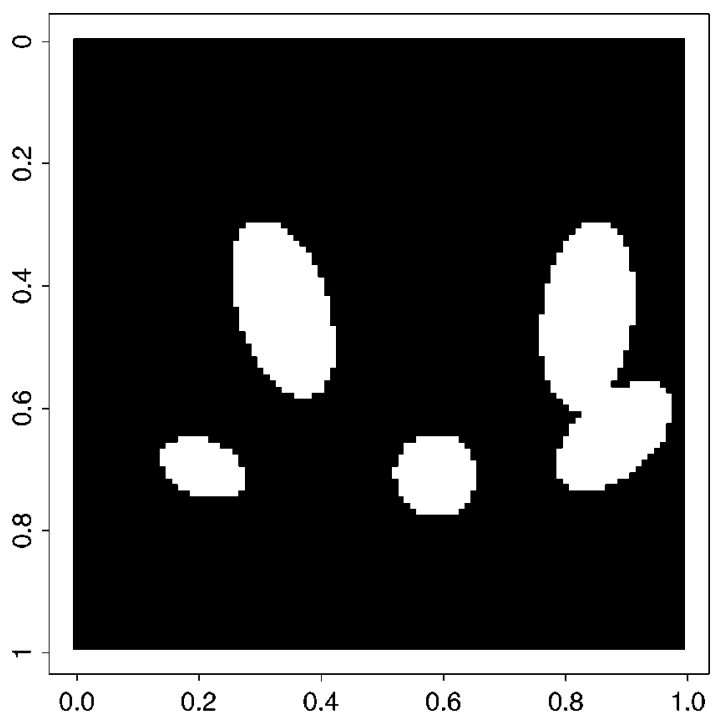

To carry out our analysis, we prepare a single-stage Hough Transform which makes use of the space curve for center position and area:

$$
\left(x_{0}(\lambda), y_{0}(\lambda), \text { area }(\lambda) ; \vec{e}_{i}, \vec{e}_{j}\right)=\left(x_{0}(\lambda), y_{0}(\lambda), \pi \rho_{1}(\lambda) \rho_{2}(\lambda)\right)
$$

where the functions $x_{0}, y_{0}$ are given in (8) and the functions $\rho_{1}$ and $\rho_{2}$ are given in (12).

We prepared a $100 \times 100 \times 100$ accumulator array whose axes were center position in the unit square and area between 0 and 0.04 , initialized the array, and carried out our simple voting process in exactly the same manner described in the two-dimensional problems described above. The resulting list of local maxima appears in Table 1.

\section{CONCLUSIONS}

In this paper, we have described a new family of single-stage Hough Transforms for detecting any collection of ellipse parameters or characteristics computable from those parameters. We have also indicated how a priori information can be explicitly included in the voting procedure. We have demonstrated the flexibility of these tools by carrying out simple experiments to detect area and orientation information and to detect center position and area information. Furthermore, we have offered our software on the Internet in an object-oriented format for the public to evaluate these techniques in conjunction with many other well-studied computational techniques.

We expect these parameter curve techniques to provide customized algorithms for those investigating specific properties of ellipse fields and to provide additional tools for those pursuing the full five-parameter ellipse detection problem.

\section{ACKNOWLEDGMENT}

Our software has been posted at http://computer.org/tpami/.

\section{REFERENCES}

[1] J. Cabrera and P. Meer, "Unbiased Estimation of Ellipses by Bootstrapping," IEEE Trans. Pattern Analysis and Machine Intelligence, vol. 18 , no. 7, pp. 752-756, July 1996.

[2] J. Canny, "A Computational Approach to Edge Detection," IEEE Trans. Pattern Analysis and Machine Intelligence, vol. 8, no. 6, pp. 679-698, 1986.

Fig. 8. A collection of ellipsoidal shapes with various areas and orientations. 
[3] J. Hall, M. Ponzi, M. Gonfalini, and G. Maletti, “Automatic Extraction and Characterization of Geological Features and Textures From Borehole Images and Core Photographs," Trans. SPWLA 37th Ann. Logging Symp., 1996.

[4] P.V.C. Hough, "Method and Means for Recognizing Complex Patterns," U.S. Patent 3,069,654, 18 Dec. 1962.

[5] J. Illingworth and J. Kittler, "A Survey of the Hough Transform," Computer Vision, Graphics, Image Processing, vol. 44, pp. 87-116, 1988.

[6] H. Kalviainen and P. Hirvonen, "An Extension of the Randomized Hough Transform Exploiting Connectivity," Pattern Recognition Letters, vol. 18, no. 1, pp. 77-85, 1997.

[7] R. Kirsch, "Computer Determination of the Constituent Structure of Biological Images," Computers and Biomedical Res., vol. 4, pp. 315-328, 1971.

[8] V.F. Leavers, "The Dynamic Generalized Hough Transform: Its Relationship to the Probabilistic Hough Transforms and an Application to the Concurrent Detection of Circles and Ellipses," CVGIP: Image Understanding, vol. 56, no. 3, pp. 381-398, Nov. 1992.

[9] E. Lyvers and O.R. Mitchell, "Precision Edge Contrast and Orientation Estimation," IEEE Trans. Pattern Analysis and Machine Intelligence, vol. 10, no. 6, pp. 23-28, 1988.

[10] S. Mallat and S. Zhong, "Characterization of Signals From Multiscale Edges," IEEE Trans. Pattern Analysis and Machine Intelligence, vol. 14, no. 7, pp. 710-732, July 1992.

[11] D. Marr and E. Hildreth, "Theory of Edge Detection," vol. 207, series B, pp. 187-217, Proc. Royal Soc. London, 1980.

[12] E.A. Maxwell, The Methods of Plane Projective Geometry Based on the Use of General Homogeneous Coordinates. Cambridge, England: Cambridge Univ. Press, 1946.

[13] H. Muammar and M. Nixon, "Tristage Hough Transform for Multiple Ellipse Extraction," IEE Proc.-E, vol. 138, no. 1, pp. 27-35, Jan. 1991.

[14] D. Scott, "Average Shifted Histograms: Effective Nonparametric Density Estimators in Several Dimensions," Annals of Statistics, vol. 13, no. 3, pp. 1,024-1,040, 1985.

[15] D. Shaked, O. Yaron, and N. Kiryati, "Derived Stopping Rules for the Probabilistic Hough Transform by Sequential Analysis," Computer Vision and Image Understanding, vol. 63, no. 3, pp. 512-526, 1996.

[16] M. Soffer and N. Kiryati, "Guaranteed Convergence of the Hough Transform," Computer Vision and Image Understanding, vol. 69, no. 2, pp. 119-134, 1998.

[17] H. Tsukune and K. Goto, "Extracting Elliptical Figures From an Edge Vector Field," IEEE Computer Vision and Pattern Recognition Conf., New York, 1983.

[18] H. Yuen, J. Illingworth, and J. Kittler, "Ellipse Detection Using the Hough Transform," AVC88: Proc. Fourth Alvey Vision Conf., 1988.

[19] D. Ziou and A. Koukam, "Knowledge-Based Assistant for the Selection of Edge Detectors," Pattern Recognition, vol. 31, no. 5, pp. 587-596, 1998. 